\title{
Acute Myocardial Infarction in a 26-Year-Old Patient With Familial Hypercholesteremia
}

\author{
Takeshi Miyayama ${ }^{a}$, Shin-ichiro Miura ${ }^{\text {b, c, d }}$, Tomo Komaki ${ }^{b}$, Takashi Kuwano ${ }^{\text {, J Joji Morii }}{ }^{b}$, \\ Hiroaki Nishikawa ${ }^{\text {, }}$ Keijiro Saku ${ }^{\text {b, }}$
}

\begin{abstract}
A 26-year-old male suffered sustained chest pain. Electrocardiogram showed ST-segment elevation in the anteroseptal wall and reciprocal ST-segment change in the inferior wall. The troponin-I level and the white blood cell count were elevated. We gave a diagnosis of acute myocardial infarction. He underwent urgent coronary angiography, which revealed $90 \%$ diffuse stenosis in the middle right coronary artery and total occlusion in the proximal left anterior descending coronary artery (LAD). Since the electrocardiogram indicated that the culprit lesion was in the proximal LAD, we performed percutaneous coronary intervention. The coronary flow in the LAD was classified as thrombolysis in myocardial infarction trial 3 . His coronary risk factors were obesity, smoking, family history, hypertension and diabetes, in addition to heterozygous familial hypercholesteremia (FH). Herein, we describe the case of a young patient with acute anteroseptal myocardial infarction and discuss the potential importance of controlling cholesterol levels in FH.
\end{abstract}

Keywords: Acute myocardial infarction; Percutaneous coronary intervention; Familial hypercholesteremia

\section{Introduction}

Familial hypercholesterolemia (FH) is the most important and commonly inherited cause of premature coronary artery disease (CAD) [1]. Heterozygosity is observed in 1 in every 500 people, and homozygosity is seen in approximately 1 in every

Manuscript accepted for publication May 17, 2016

aPostgraduate Clinical Training Center, Fukuoka University Hospital, Fukuoka, Japan

${ }^{b}$ Department of Cardiology, Fukuoka University School of Medicine, Fukuoka, Japan

'Department of Molecular Cardiovascular Therapeutics, Fukuoka University School of Medicine, Fukuoka, Japan

${ }^{\mathrm{d} C o r r e s p o n d i n g ~ A u t h o r: ~ S h i n-i c h i r o ~ M i u r a, ~ D e p a r t m e n t ~ o f ~ C a r d i o l o g y, ~ F u k u o-~}$ ka University School of Medicine, 7-45-1 Nanakuma, Jonan-ku, Fukuoka 814-0180, Japan.Email: miuras@cis.fukuoka-u.ac.jp

doi: http://dx.doi.org/10.14740/jocmr2596w
1 million people in Japan [2]. A diagnosis of FH can be made clinically (by history, clinical examination and measurement of serum cholesterol levels) or by DNA analysis. According to the Japan Atherosclerosis Society (JAS) Guidelines in 2012, diagnostic criteria for heterozygous $\mathrm{FH}$ are two or more of the following: 1) low-density lipoprotein cholesterol (LDL-C) > $180 \mathrm{mg} / \mathrm{dL} ; 2$ ) tendon/skin xanthoma(s); and 3) family history of $\mathrm{FH}$ or premature $\mathrm{CAD}$ within second-degree relatives [3].

Increasing age, elevated levels of total cholesterol (TC) and LDL-C, low levels of high-density lipoprotein cholesterol (HDL-C), male gender, smoking, metabolic syndrome, diabetes, hypertension, and a family history of early CAD are risk factors for the development of CAD. These factors accelerate the development of atherosclerosis in patients with and without FH and must be treated aggressively, especially in patients with FH [4]. This case report highlights the potential importance of controlling cholesterol levels to prevent CAD in patients with heterozygous FH.

\section{Case Report}

A 26-year-old male complained of chest pain. He had begun to feel easily fatigued over the previous 2 weeks. Chest pain was sustained for $5 \mathrm{~min}$ and also appeared twice when he was at rest watching TV. On the day of presentation, the chest pain appeared suddenly during work. Since the symptom was sustained, he went to the nearest clinic. ST elevation was observed in $\mathrm{V}_{1-5}$ of a 12-lead electrocardiogram, and he was referred to our hospital due to suspected acute myocardial infarction. At the time of emergency transportation, his level of consciousness was good. Although his blood pressure was 153/98 mm $\mathrm{Hg}$, other vital signs were normal. There were otherwise no obvious abnormal findings in the physical examination.

With regard to biochemical parameters in blood, the creatine phosphokinase level, renal, liver and coagulative functions and the C-reactive protein level were all normal, whereas the troponin-I level $(98.9 \mathrm{pg} / \mathrm{mL})$ and the white blood cell count $(14,000 / \mu \mathrm{L})$ were elevated. Serum levels of TC, LDL-C, HDL-C and triglyceride (TG) were 218, 151, 50 and $40 \mathrm{mg}$ / $\mathrm{dL}$, respectively. Chest radiography showed cardiac dilatation (cardiothoracic ratio was 53\%), whereas there was no plural effusion or pulmonary congestion. The electrocardiogram showed ST-segment elevation in the anteroseptal wall $\left(\mathrm{V}_{1-4}\right)$, 


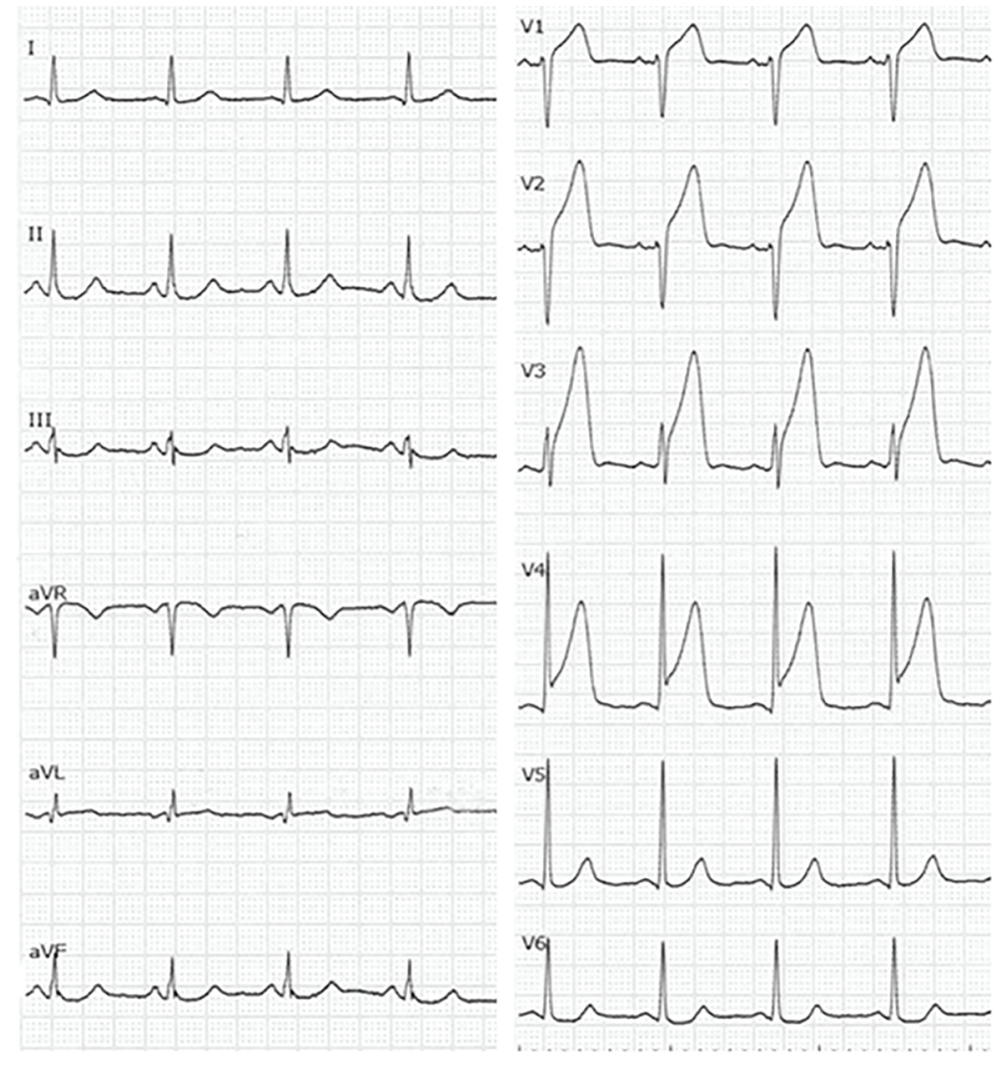

Figure 1. Electrocardiogram at admission.

and reciprocal ST-segment change in the inferior wall (II, III and aVf) (Fig. 1). Thoracic echocardiogram showed asynergic motion in the anteroseptal wall from the base to the apex. Based on these findings, we gave a diagnosis of acute anteroseptal myocardial infarction. He underwent urgent coronary angiography, which revealed total occlusion in the proximal left anterior descending coronary artery (LAD) (segment 7) and $90 \%$ diffuse stenosis in the middle right coronary artery (RCA) (segments 2-3) (Fig. 2). Since the electrocardiogram indicated that the culprit lesion was in the proximal LAD, we performed percutaneous coronary intervention (PCI) (stent implantation (Promus PREMIER $4.0 \times 16 \mathrm{~mm}$ )). The coronary flow in the LAD was classified as thrombolysis in myocardial infarction trial 3.

A medical check-up 1 year previously had noted obesity (body mass index $26.7 \mathrm{~kg} / \mathrm{m}^{2}$ ), high blood pressure $(150 / 96$ $\mathrm{mm} \mathrm{Hg}$ ), abnormal lipid profile (TC, LDL-C and TG were 245, 172 and $163 \mathrm{mg} / \mathrm{dL}$, respectively), and diabetes (hemoglocbinA1c (NGSP) $6.6 \%$ and fasting blood glucose $130 \mathrm{mg} / \mathrm{dL}$ ). Additionally, he smoked 20 cigarettes a day. Figure 3 shows

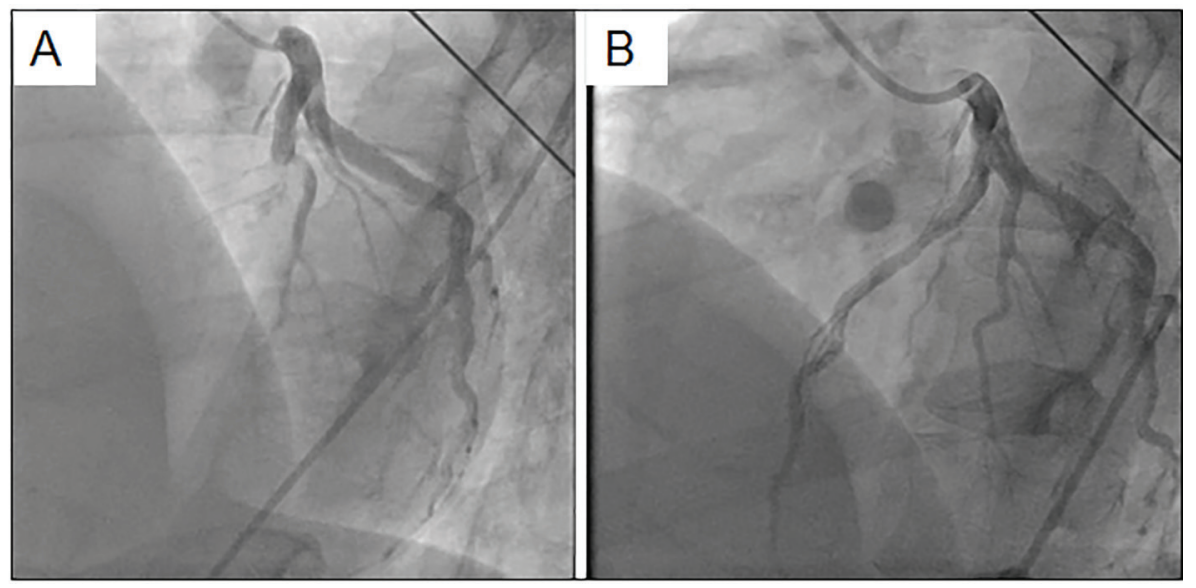

Figure 2. Coronary angiography in the left coronary artery before (A) and after (B) coronary intervention. 


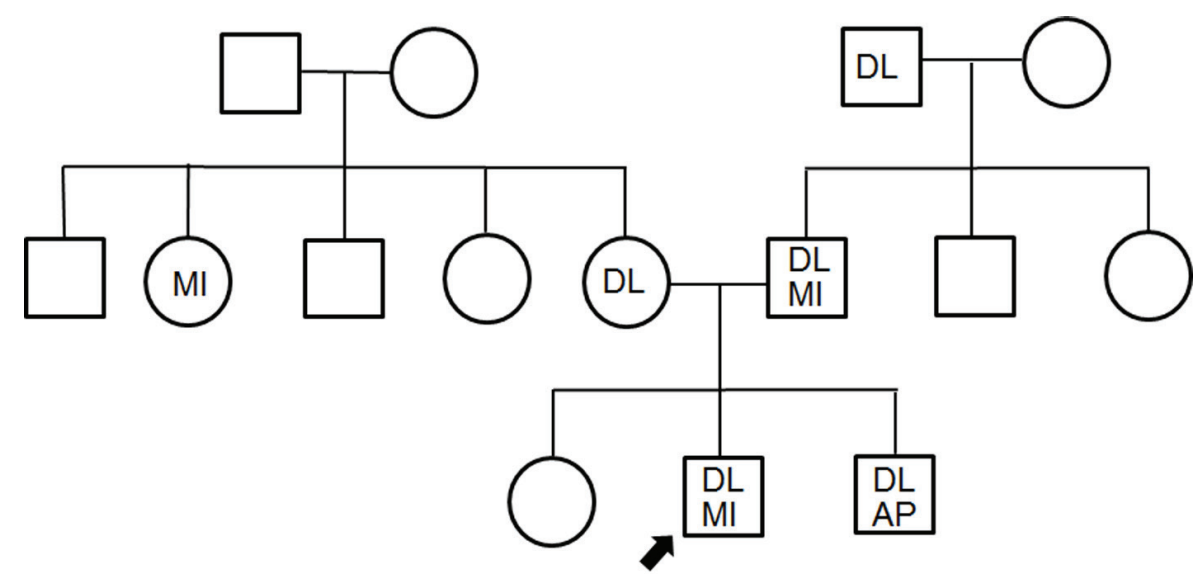

Figure 3. Pedigree chart with third-degree relatives. DL: dyslipidemia; MI: myocardial infarction; AP: angina pectoris. The squares and circles indicate males and females.

a pedigree chart with third-degree relatives. His grandfather, parents and older brother all had a history of dyslipidemia. His older brother and father had received PCI for CAD at ages of 33 and 48 years, respectively. In addition, he had skin and tendon xanthomata (Fig. 4) without corneal arcus. Since a clinical diagnosis of heterozygous FH is based on high cholesterol levels in combination with a family history of hypercholesterolemia, premature $\mathrm{CAD}$, and/or physical examination findings of xanthomata, he was clinically diagnosed as heterozygous FH. His coronary risk factors were obesity, smoking, family history, hypertension and diabetes mellitus, in addition to heterozygous $\mathrm{FH}$.

After PCI, there was no condition of heart failure, although the peak CPK was 5,599 U/L. After cardiac rehabilitation for approximately 3 weeks, we performed again PCI for the RCA lesion before discharge (segment 2: stent implantation (Ultimaster $3.5 \times 33 \mathrm{~mm}$ ); segment 3: balloon dilatation). He received angiotensin converting enzyme inhibitor and $\beta$-blocker. Intensive lipid-lowering therapy (atorvastatin $20 \mathrm{mg}$ /day) was performed and the LDL-C level was $72 \mathrm{mg} / \mathrm{dL}$. Finally, he was discharged after 22 days of hospitalization without the recurrence of any symptoms.

\section{Discussion}

We experienced a case of acute myocardial infarction in a young patient with heterozygous $\mathrm{FH}$. FH itself is a significant $\mathrm{CAD}$ risk factor because it results in long-term exposure to high lipid levels [5]. The risk for the development of CAD in patients with untreated heterozygous FH is 20 times higher than that in treated patients. Thus, multidetector-row computed tomography should be performed early to identify coronary stenosis when CAD is suspected in patients with heterozygous FH. In this case, the patient had many risk factors including smoking, in addition to FH. Smoking is an extremely important and modifiable risk factor in patients with $\mathrm{FH}$, and must be avoided due to the increased likelihood of the development of very premature-onset CAD [6].

Statins are used as first-line drugs in patients with FH.
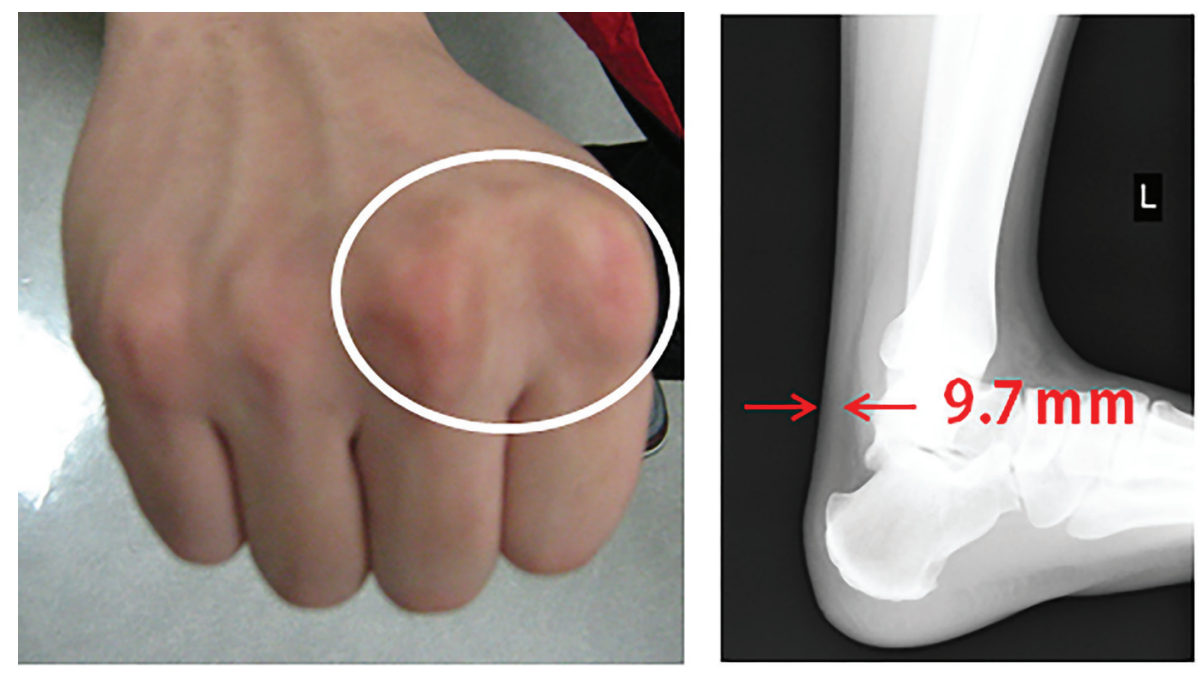

Figure 4. Skin and tendon xanthoma. 
Recently, Rallidis et al reported that one of five patients who develop very early ST-segment elevation myocardial infarction at $\leq 35$ years of age has definite/probable heterozygous $\mathrm{FH}$ and, despite the use of statins, there is a therapeutic gap and a high recurrence rate of cardiac events during long-term follow-up [7]. Although the LDL-C level for secondary prevention and $\mathrm{FH}$ patients is less than $100 \mathrm{mg} / \mathrm{dL}$ according to the JAS Guidelines of 2012, patients with FH must be treated under the guidance of a specialist. In this respect, an additional therapeutic option is now available, which entails the use of proprotein convertase subtilisin/kexin 9 (PCSK-9) inhibitors [8]. PCSK-9 inhibitors inhibit the binding of PCSK-9 with LDL receptors and prevent the degradation of LDL receptors. These inhibitors have excellent lipid-lowering properties and may have a beneficial effect on the CAD outcome in patients with $\mathrm{FH}[9]$.

In conclusion, this case emphasizes the need to clinically recognize xanthomas and the familial inheritance of $\mathrm{FH}$ with elevated LDL-C. Early diagnosis and the early initiation of treatment can save the affected individual and other family members. In addition, FH should be suspected in premature CAD patients with high LDL-C levels.

\section{Conflicts of Interest/Disclosures}

K.S. is a Chief Director and S.M. is a Director of NPO Clinical and Applied Science, Fukuoka, Japan. K.S. has an Endowed "Department of Molecular Cardiovascular Therapeutics" supported by MSD, Co. LTD. S.M. belongs to the Department of Molecular Cardiovascular Therapeutics supported by MSD, Co. LTD.

\section{References}

1. Risk of fatal coronary heart disease in familial hypercholesterolaemia. Scientific Steering Committee on behalf of the Simon Broome Register Group. BMJ. 1991;303(6807):893-896.
2. Yu W, Nohara A, Higashikata T, Lu H, Inazu A, Mabuchi $\mathrm{H}$. Molecular genetic analysis of familial hypercholesterolemia: spectrum and regional difference of LDL receptor gene mutations in Japanese population. Atherosclerosis. 2002;165(2):335-342.

3. Harada-Shiba M, Arai H, Oikawa S, Ohta T, Okada T, Okamura T, Nohara A, et al. Guidelines for the management of familial hypercholesterolemia. J Atheroscler Thromb. 2012;19(12):1043-1060.

4. Goldberg AC, Hopkins PN, Toth PP, Ballantyne CM, Rader DJ, Robinson JG, Daniels SR, et al. Familial hypercholesterolemia: screening, diagnosis and management of pediatric and adult patients: clinical guidance from the National Lipid Association Expert Panel on Familial Hypercholesterolemia. J Clin Lipidol. 2011;5(3 Suppl):S1-8.

5. Alonso R, Mata N, Castillo S, Fuentes F, Saenz P, Muniz $\mathrm{O}$, Galiana J, et al. Cardiovascular disease in familial hypercholesterolaemia: influence of low-density lipoprotein receptor mutation type and classic risk factors. Atherosclerosis. 2008;200(2):315-321.

6. Neil A, Cooper J, Betteridge J, Capps N, McDowell I, Durrington P, Seed M, et al. Reductions in all-cause, cancer, and coronary mortality in statin-treated patients with heterozygous familial hypercholesterolaemia: a prospective registry study. Eur Heart J. 2008;29(21):2625-2633.

7. Rallidis LS, Triantafyllis AS, Tsirebolos G, Katsaras D, Rallidi M, Moutsatsou P, Lekakis J. Prevalence of heterozygous familial hypercholesterolaemia and its impact on long-term prognosis in patients with very early STsegment elevation myocardial infarction in the era of statins. Atherosclerosis. 2016;249:17-21.

8. Lepor NE, Kereiakes DJ. The PCSK9 Inhibitors: A Novel Therapeutic Target Enters Clinical Practice. Am Health Drug Benefits. 2015;8(9):483-489.

9. Stroes E, Colquhoun D, Sullivan D, Civeira F, Rosenson RS, Watts GF, Bruckert E, et al. Anti-PCSK9 antibody effectively lowers cholesterol in patients with statin intolerance: the GAUSS-2 randomized, placebo-controlled phase 3 clinical trial of evolocumab. J Am Coll Cardiol. 2014;63(23):2541-2548. 